\title{
Imaging polarimetry for the determination of stress constraint in transparent solids
}

\author{
M. Richert ${ }^{1}$, X. Orlik ${ }^{1}$, and A. De Martino ${ }^{2}$ \\ ${ }^{1}$ ONERA, the Theoretical and Applied Optics Department (DOTA), 31055 Toulouse Cedex 4, France \\ ${ }^{2}$ LPICM, UMR 7647, Ecole polytechnique, 91128 Palaiseau
}

\begin{abstract}
Using a Mueller matrix polarimeter based on liquid crystal cells [1] and the forward polar decomposition [2], we study the evolution of the birefringence property induced by an increasing compression of a Plexiglas sample. We measure the 2D distribution of the relative retardation $\delta$, the azimuth angle $\alpha$ and the ellipticity angle $\varepsilon$ of the sample. A noticeable advantage of such method is the possibility to map the stressinduced birefringence even if the material used for the reflexion implies partial depolarization of the scattered field. More generally, this method will be particularly useful in experimental situations where the light beam undergoes depolarization and/or dichroïsm in addition to birefringence effects because classical birefringence measurement methods are not able to perform an efficient discrimination between these 3 effects. Our experiment shows that the Plexiglas sample under investigation exhibits some weak stress-induced elliptic birefringence. According to simple numerical simulations, this observation is explained by the use of a bi-static illumination/detection configuration.
\end{abstract}

\section{Introduction}

There is considerable interest in spatially quantifying the parameters describing optical anisotropy [3-7]. Many living biological specimens [4-8] as well as photonic devices [8-10] exhibit low-level birefringence eventually induced by stresses. For instance, the determination of stresses in transparent thin plates (or films) plays an important role in optoelectronic technologies.

Several techniques for determining the optical birefringence have been used including compensatorbased methods $[7,11,12]$, interferometric setups $[3,6,8$, $13]$, and mostly polarimetric experiments $[4,5,14]$.

In order to perform a complete polarimetric characterization of an unknown sample, we need to acquire its Mueller Matrix. For such measurement, we use Mueller matrix polarimeters composed of a polarization-state generator (PSG) and a polarizationstate analyser (PSA) that respectively generates and analyses the light using four linearly independent states. The 16 elements of this matrix characterize the 3 polarimetric properties driving amplitude and/or phase relations between 2 orthogonal polarization states of the electric field scattered by the sample : the birefringence (or retardance) that traduces a dephasing, the diattenuation (or dichroism) that describes a differential attenuation and the depolarization that highlights the phase breaks provoked mainly by multiple scattering.

However, only the diattenuation properties can be directly obtained from the Mueller matrix. The two other properties can be reached in the general case only using a decomposition algorithm. In this work, we perform the forward polar decomposition developed by $\mathrm{Lu}$ and Chipman [2].

As a first step, we present the Mueller polarimeter we have developed and characterize the instrument accuracy using 2 reference samples: a polarizer and a retarder plate. As a second step, we use our setup to study a Plexiglas sample exhibiting some stress-induced birefringence in a backscaterring configuration.

\section{Experimental setup and calibration}

\subsection{The Mueller polarimeter}

The Mueller matrix polarimeter is composed of a Polarization State Generator (PSG) that consists of a Glan-Thompson polarizing prism followed by 2 nematic liquid crystals (from Meadowlark optics) acting as adjustable retardance elements and of a Polarisation State Analyser (PSA) that includes the same components, but with the prism placed before the nematic liquid crystals

This is an Open Access article distributed under the terms of the Creative Commons Attribution-Noncommercial License 3.0, which permits unrestricted use, distribution, and reproduction in any noncommercial medium, provided the original work is properly cited. 
(figure 1). Our light source is a Nd-Yag doubled continuous laser (from Spectra Physics) that operates at $532 \mathrm{~nm}$. The values of the orientation and retardance of each of the 4 dephasing elements are chosen in order to minimize the propagation of errors from intensities to the calculus of Mueller matrices [15, 16].

The polarimeter works in backscattering configuration. The full-angle between the emission and detection arm is about $10^{\circ}$, and the sample is at $1.5 \mathrm{~m}$ from the laser.

The Mueller matrix of the sample is obtained by generating successively four linearly independent states of polarization contained in a $4 \times 4$ matrix called $\mathbf{W}$ (each column represents a Stokes vector), and by analysing the backscattering field projected along four linearly independent states of polarization contained in a $4 \times 4$ matrix called A (each lines representing the Stokes vector of the state of analysis). Let the 16 intensity measurements be defined by the matrix I. Thus if we know the matrices $\mathbf{A}$ and $\mathbf{W}$ we can calculate the Mueller matrix of the sample as follow:

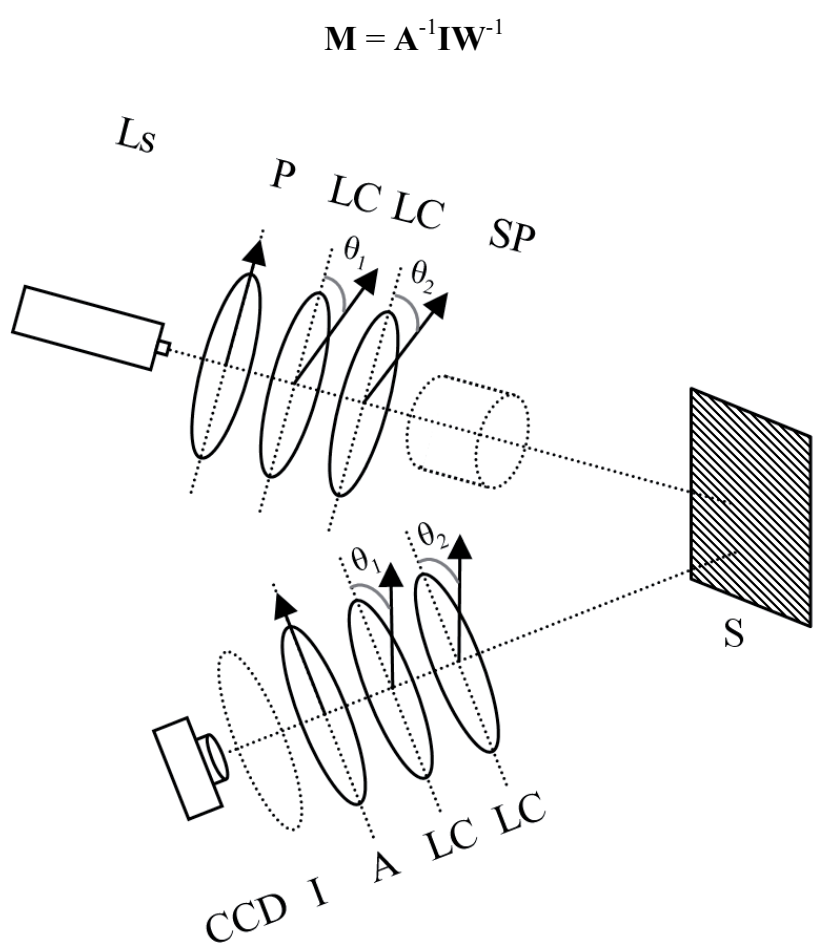

Fig. 1. Schematic of the imaging polarimeter in the reflexion configuration setup. LS, laser source $532 \mathrm{~nm}$; P, linear polarizer with transmission axis indicated by arrow; LC, liquid-crystal variable retarder with fast axis direction indicated by arrow; SP, spatial filter; S, sample; A, linear analyzer with transmission axis indicated by arrow; I, imaging lens; CCD, CCD camera.

One of the most critical issue of the measurement of a Mueller matrix sample is the precise estimation of the two modulation matrices $\mathbf{A}$ and $\mathbf{W}$. In order to determine these matrices we have used the eigeinvalues calibration method (ECM) developed by Compain [17]. The main advantages of this method is that there is no need to know the precise orientation of the elements. Furthermore, it takes into account the numerous inherent defects of the optical elements that constitute the PSA and the PSG.

\subsection{Accuracy measurement of the polarimeter}

To evaluate the overall system performance, we've taken a serie of Mueller matrix measurements on a sandblasted aluminium plate placed after a Glan-Thompson polarizer used at different azimuthal angles. A typical result at 532 $\mathrm{nm}$ is shown in Figs. 2 for the average over $250 \times 250$ pixels. One this figure the dot curves represent experimental values and the solid ones represent the elements of the Mueller matrices obtained by the multiplication of the experimental matrix of the aluminium plate by the theoretical Mueller matrix of a perfect polarizer. All the data have been normalized by the $\mathrm{M}_{00}$ elements of the Mueller matrix. The mean deviation between "pseudo" theoretical and experimental values is about 0.02 and always smaller than 0.05 .

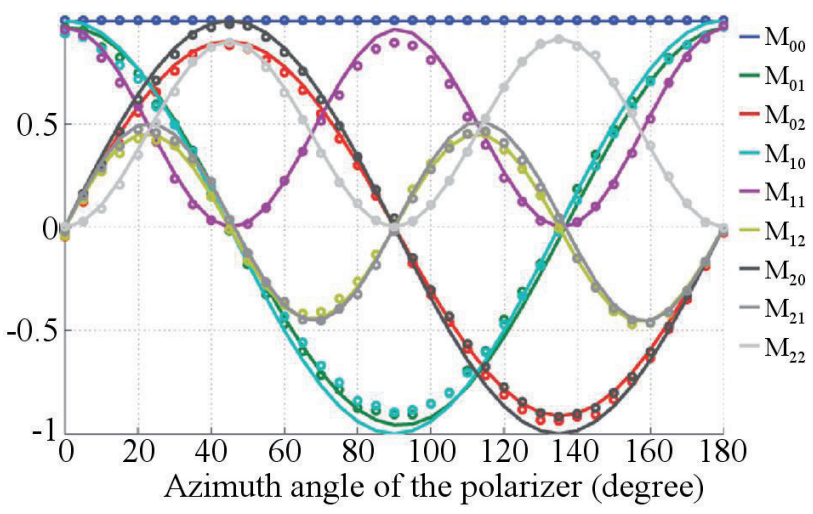

Fig. 2. Mueller matrix measurements in function of the azimuth angle of the polarizer: dot curves represent experimental values and the solid ones represent the elements of the Mueller matrices obtained by the multiplication of the experimental matrix of the aluminium plate by the theoretical Mueller matrix of a perfect polarizer (a): polarizer

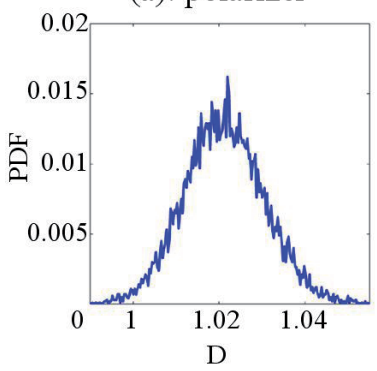

(b): retarder plate

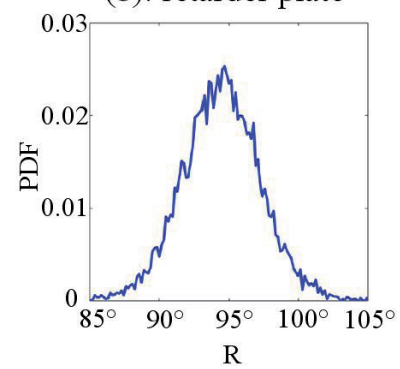

Fig. 3. Estimation of the accuracy of the Lu \& Chipmann decomposition combine with the polarimeter for the determination of the diattenuation of a polarizer (a) and the retardance of a retarder plate $(b)$

Then, using the polar decomposition, another evaluation of the accuracy of our setup has been performed by determining the diattenuation and the retardance of respectively a Glan-Thompson polarizer and a quarter-wave plate (at $532 \mathrm{~nm}$ ). On figure 3 is plotted the probability density function of these two parameters over an image $(250 \times 250$ pixels $)$. The Gaussian shape of these two distributions is due to the 
speckle noise inherent to the use of the coherent source. Concerning the diattenuation of the polarizer, we have obtained a mean value of 1.02 and a standard deviation of 0.012 , and for the retardance of the quarter-wave plate ${ }^{\mathrm{a}}$, we have measured a mean value of $94.44^{\circ}$ with a standard deviation of $3.80^{\circ}$.

\section{Results and interpretation}

\subsection{Experimental setup}

To analyse the stress-induced birefringence in a transparent solid with the polarimeter in a backscattering configuration, a sandblasted aluminium plate is used to reflect the laser light just after its propagation through a Plexiglas plate $(40 \times 40 \mathrm{~mm})$ that is increasingly compressed using five screws according to the figure 4. The polarimetric image of the Plexiglas plate is formed into a CCD camera through a zoom lens. For each state of polarization of the PSA and PSG, 20 images of the sample are performed in order to reduce the detection noise. After measuring the Mueller matrix for each situation of compression, the forward polar decomposition is used to split this matrix into a product of 3 elementary matrices: a diattenuator followed by a retarder and then followed by a depolarizer matrix. From the retarder matrix, and for each pixel of the CCD camera, we extract the 3 degrees of freedom of the birefringence property: the scalar retardance $R$, the azimuth $\alpha$ and the ellipticity $\varepsilon$ of the retardance vector.
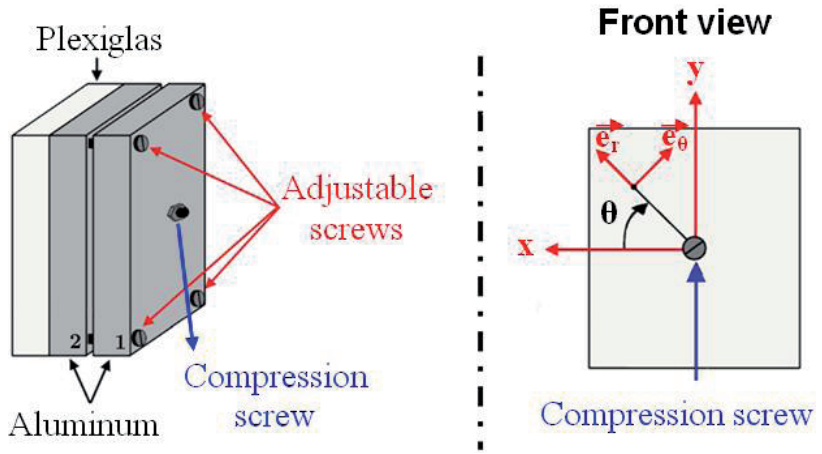

Fig. 4. Schematic view of the compression system

As we work on reflexion, we have taken great care about the convention of the coordinate system in which the properties of polarization are expressed. If we make the hypothesis that for each propagation of the round trip on the aluminium plate, the Plexiglas is a linear retarder with a fixed orientation, then by using the usual convention, the calculation shows that the system (Aluminium/Plexiglas) behaves, after this round trip, as an elliptic retarder, with an orientation and a scalar retardance described by rather complicated equations. Thus, we have chosen to work with the Jones convention. In this latter coordinate system, the system then behaves after a round trip on the aluminium plate, as a linear retarder with the same orientation as for a single trip, and

${ }^{a}$ Quarter-wave plate at $532 \mathrm{~nm}$ measured with a laser source at $514.5 \mathrm{~nm}$, thus $\mathrm{R}=93.03^{\circ}$ with a scalar retardance simply equal to twice the value of a single trip.

\subsection{Birefringent measurements}

Performing the polar decomposition, we have measured the scalar retardance and the orientation (azimuth and ellipticity) of the birefringence in function of the torque strength of the four adjustable screws (figure 4).

On figure 5 is presented the scalar retardance of the system: \{Plexiglas-Aluminum\} for four different torques strengths: $0.00,0.44,0.87$ and 1.25 N.m.

As expected, the images of the retardance exhibit approximately a cylindrical symmetry along the screw axis and the scalar retardance increases as we approach the screw. However for high values of the torque strength (figure 5.c and 5.d), we observe a folding of the retardance that comes from the fact that the Mueller matrix of a retarder is defined for a scalar retardance between 0 and $\pi$.

Concerning the azimuth of the retardance vector, as for the retardance image, we observe a cylindrical symmetry. However, we can notice some discontinuities along the $\mathrm{x}$ and $\mathrm{y}$ axis and at the border of each ring. The horizontal and vertical discontinuities are due to the mathematical definition of the orientation of the retarder (a vertical retarder can be described with an azimuth at $+\pi$ or $-\pi)$. The second types of discontinuities come from the same cause as for the scalar retardance folding. For instance, if the retardance $\mathrm{R}$ becomes greater than $\pi$ (and remains smaller than $2 \pi$ ), there is a permutation between the fast and slow axis and the "new" retardance R' will be equal to $\pi$-R.

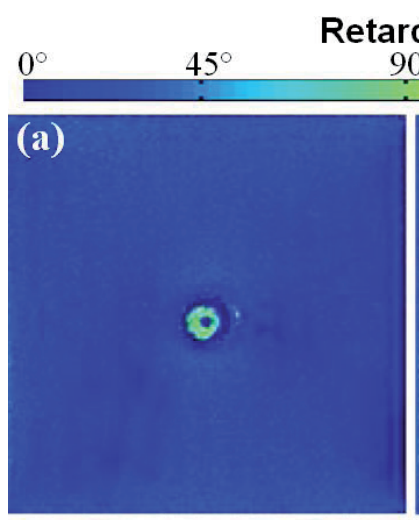

Retardance 135 $180^{\circ}$
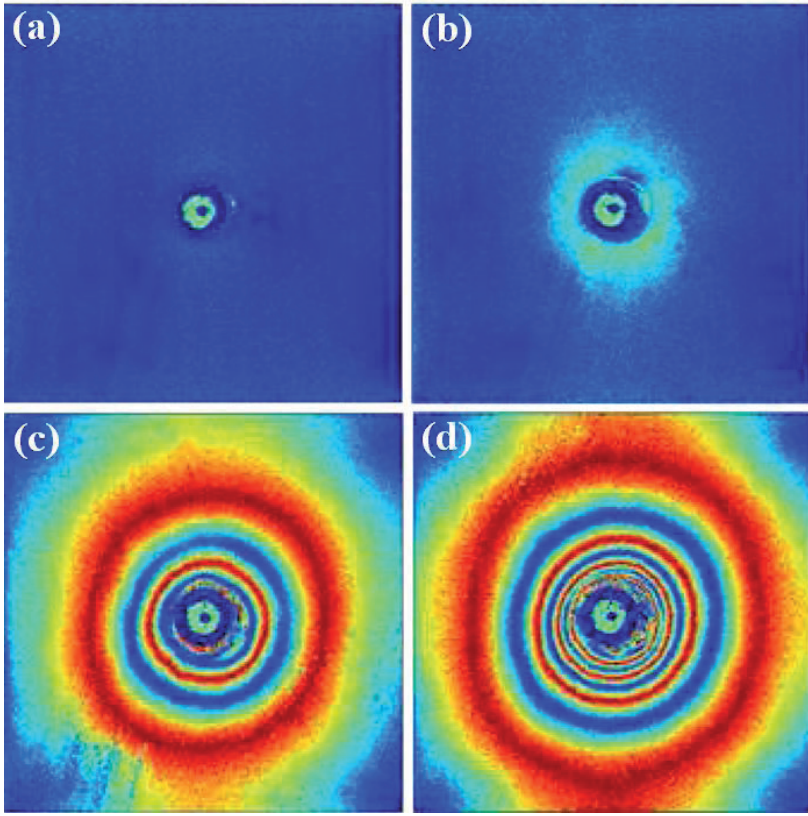

Fig. 5. Retardance images of the system in function of the torque strength. (a): 0.00 N.m, (b): 0.44 N.m, (c): 0.87 N.m and (d): 1.25 N.m 


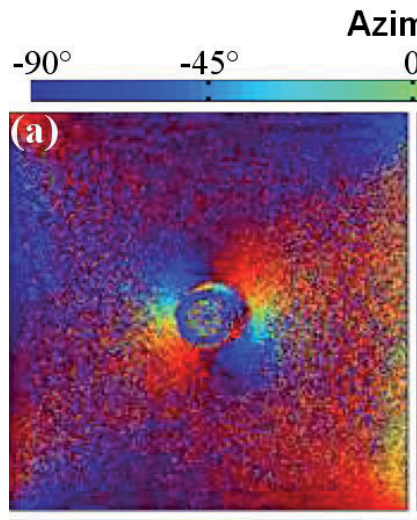

Azimuth

$0^{\circ} \quad 45^{\circ} \quad 90^{\circ}$
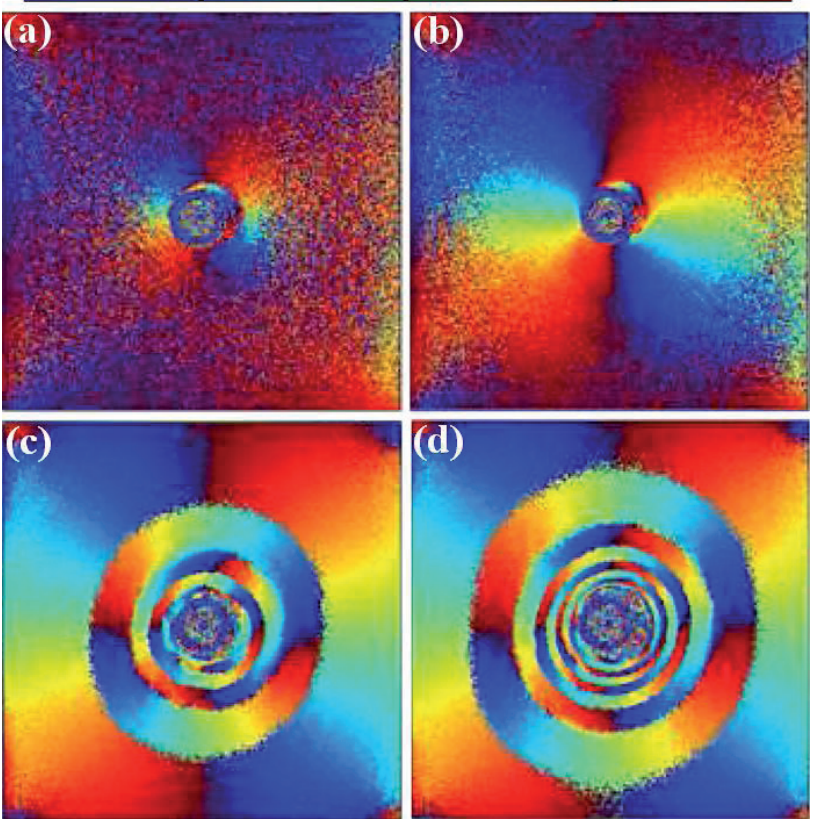

Fig. 6. Azimuth of the retardance vector of the system in function of the torque strength. (a): $0.00 \mathrm{~N} . \mathrm{m}$, (b): 0.44 N.m, (c): 0.87 N.m and (d): 1.25 N.m

\begin{tabular}{cccc} 
& \multicolumn{4}{c}{ Ellipticity } \\
$-45^{\circ}$ & $-22.5^{\circ}$ & $0^{\circ}$ & $22.5^{\circ}$ \\
\hline
\end{tabular}
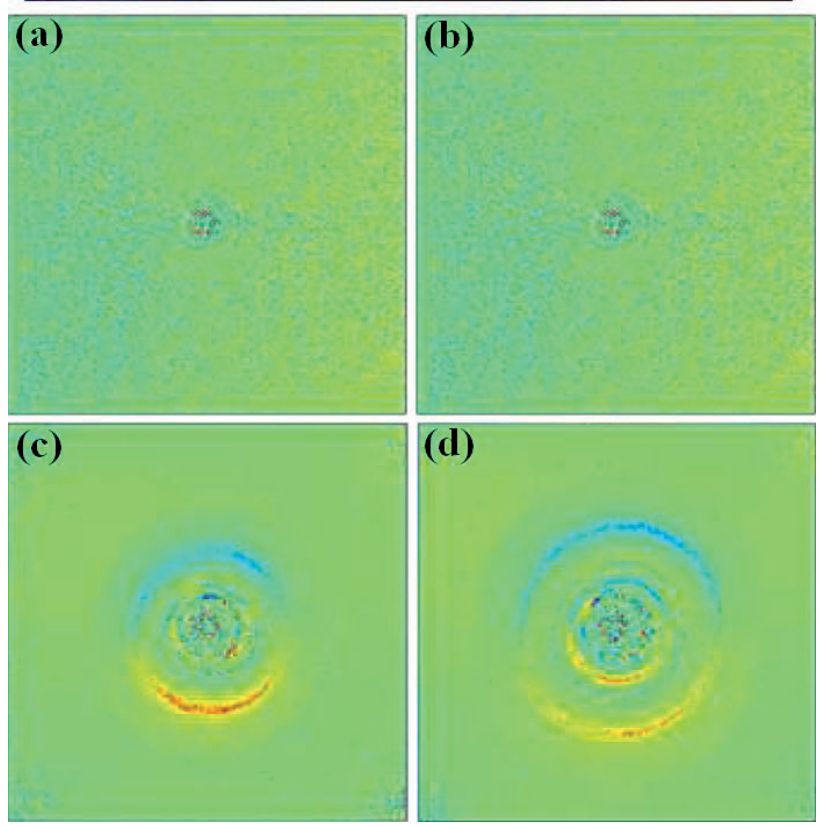

Fig. 7. Ellipticity of the retardance vector of the system in function of the torque strength. (a): $0.00 \mathrm{~N} . \mathrm{m}$, (b): 0.44 N.m, (c): 0.87 N.m and (d): 1.25 N.m

The last series of images concern the ellipticity $\varepsilon$ of the retardance vector of the system. For low torque strength (figure 7.a and figure 7.b), the system behaves approximately as a linear retarder $(\varepsilon=0)$. However, for high torque strength we can notice non negligible ellipticity in some area of the image. Moreover, we observe some correlations between regions of high ellipticity and regions of the retardance image exhibiting near zero values. Furthermore, if we take a look at the retardance and ellipticity image along a radial direction, we can notice that for one passage by a zero value of the retardance over two, the ellipticity gets high.

\subsection{Numerical simulations}

As we use the polarimeter in a backscattering configuration with an oblique illumination, the intersection between the index ellipsoid and the wave plane isn't identical for the forward and backward propagation. Thus the polarimetric parameters of the Plexiglas can be different for the forward and backward propagation.

In fact, the measurement of high values of ellipticity in some regions of the image can be explained by the combination of two linear retarders with the same scalar retardance but with different orientations. For illustration, we have calculated the retardance and the ellipticity of a system composed by two linear retarder (with the same retardance but different orientations) in function of their scalar retardance $R$ (figure 8).

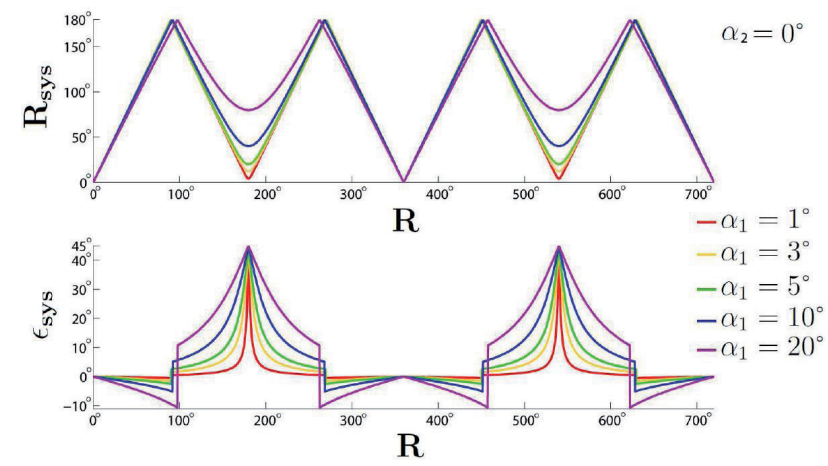

Fig. 8. Simulation of the retardance $R_{\text {sys }}$ and the ellipticity $\varepsilon_{\text {sys }}$ of a system composed by two linear retarders with the same retardance $R$ and different orientation

Each curve on the figure 8 is plotted for various differences between the two fast axes angles. We notice that as for the experimental retardance and ellipticity images, for a passage on two by a zero value of the retardance, the ellipticity gets high. We notice moreover that the peak of the ellipticity curve gets thinner with a decreasing angle between the two fast axis.

\section{Conclusion}

We have presented here a Mueller polarimeter in a backscattering configuration exhibiting accuracy around 0.02. Using the $\mathrm{Lu}$ and Chipmann decomposition, we have determined the vectorial birefringence of the system and thus visualized the associated stress constraints. The presence of elliptical birefringence has been explained by the bi-static configuration of the illumination/detection setup.

\section{References}

1. B. Laude-Boulesteix, A. De Martino, B. Drevillon, and L. Schwartz, Appl. Opt, 43, 2824-2832 (2004) 
2. S.-Y. Lu and R.A. Chipman, JOSA A Optics, Image Science and Vision, 13, 1106-1113 (1996)

3. C. K. Hitzenberger, E. Gotzinger, M. Sticker, M Pircher and A. F. Fercher, Opt. Express, 9, 780-789 (2001)

4. T. Oka and T. Kaneko, Opt. Express, 11, 1510-1519 (2003)

5. M. Shribak and R. Oldenbourg, Appl. Opt., 42, 30093017 (2003)

6. T. Colomb, F. Dürr, E. Cuche, P. Marquet, H. G. Limberger, R. P. Salathé and C. Depeursinge, Appl. Opt., 44, 4461-4469 (2005)

7. C.C. Montarou, T.K. Gaylord, B.L. Bachim, A.I. Dachevski and A. Agarwal, Appl. Opt., 45, 271-280 (2006)

8. F. El-Diasty, J. Opt. A: Pure \& Appl. Opt, 1, 197-200 (1999)

9. Roberts, K. Thorn, M. L. Michna, N. M. Dragomir, P. M. Farrell and G. W. Baxter, Opt. Lett., 27, 86-88 (2002)

10. N. M. Dragomir, G. W. Baxter and A. Roberts, IEE Proc. Optoel., 153, 217-221 (2006)

11. N.H. Hartshorne and A. Stuart, Crystals and the polarising microscope, (Edward Arnold Ltd. London, 1970).

12. C.C. Montarou and T.K. Gaylord, Appl. Opt., 43, 6580-6595 (2004)

13. Y. Ohtsuka and T. Oka, Appl. Opt., 33, 2633-2636 (1994)

14. J.G. Pickering and D.R. Boughner, Am. J. Pathol., 138, 1225-1231 (1991).

15. J.S. Tyo, Optics Letters, 25, 1198-1200 (2000)

16. D.S. Sabatke, M.R. Descour, E.L. Dereniak, W.C Sweatt, S.A. Kemme, and G.S. Phipps, Optics Letters, 25, 802-804 (2000)

17. E. Compain, S. Poirier, and Bernard Drevillon, Appl. Opt., 39, 3490-3502 (1999) 